J. Klin. Endokrinol. Stoffw. $2021 \cdot 14: 48-54$ https://doi.org/10.1007/s41969-021-00134-z Angenommen: 16. April 2021

Online publiziert: 6. Mai 2021

(๑) Der/die Autor(en) 2021

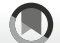

\section{Sarah Feigl}

Universitätsklinik für Frauenheilkunde und Geburtshilfe, Medizinische Universität Graz, Graz, Österreich
Hormone sind maßgeblich an Aufbau und Erhalt der Knochenmasse beteiligt. Neben Parathormon und Calcitonin nehmen Östrogen, Somatotropin und Leptin hier wesentliche Funktionen ein.

\section{Östrogen und Somatotropin}

Bei beiden Geschlechtern führt Östrogen zum Knochenwachstum und trägt zum Epiphysenschluss bei. Zu Beginn der Pubertät ist der Knochenstoffwechsel sehr aktiv. Hierzu tragen nicht nur Östrogen, sondern auch Somatotropin und InsulinLike Growth Factor 1 (IGF-1) bei.

Der pubertäre Wachstumsschub wird durch steigende Östrogenspiegel eingeleitet. Aus diesem Grund wachsen Mädchen früher als Buben, sie sind ihnen hier um etwa zwei Jahre voraus. Buben weisen erst später relevante Androgenspiegel auf, die durch Aromatisierung in Östrogen umgewandelt werden.

Östrogene stimulieren die Sekretion von Somatotropin und IGF, die ihrerseits das Längenwachstum fördern. Die Somatotropinkonzentration erreicht zum Ende der Pubertät ihren Zenit und ist nachts am höchsten. Um das volle Wachstumspotenzial auszunutzen, sind ausreichend Nachtschlaf, eine ungestörte Somatotropinsekretion und eine funktionierende Hypothalamus-HypophysenGonadenachse essenziell.

Die Wirkung des Östrogens erfolgt durch unterschiedliche Mechanismen. Zum einen steuert es die Osteoblasten, die Knochenmatrix aufbauen. Sie bilden durch Östrogeneinfluss Osteoprotegerin, das in weiterer Folge die Reifung von Osteoklasten hemmt. Zum anderen kontrolliert es die Synthese von Kollagen und Knochenproteinen (Osteokalzin und Osteopontin) sowie der alkalischen Phosphatase. Hauptsächlich hemmt Östrogen jedoch den Knochenabbau durch seinen Einfluss auf Osteoklasten durch Aktivierung von Apoptosemechanismen. Die Apoptose von Osteoblasten und Osteozyten wird wiederum durch Östrogen gehemmt. Diese Wirkungen des Östrogens werden durch Regulation unterschiedlicher Zytokinsysteme vermittelt.

Während des pubertären Wachstumsschubs ist der Knochenstoffwechsel am aktivsten, die Umbaumarker erreichen ihren Peak. Am Ende der Pubertät, wenn die Östrogenspiegel am höchsten sind, beruhigt sich der Knochenstoffwechsel, der Wachstumsschub ist vorüber $[1,2]$. Nach Eintritt der Menarche ist das Wachstum fast abgeschlossen, es verbleibt ein Wachstumspotenzial von ca. 4\% [3]. Der Epiphysenschluss besiegelt das Ende des Längenwachstums. Im Falle eines Östrogenmangels oder eines Rezeptordefektes kann dieser nicht vollständig erfolgen, das Längenwachstum wird fortgesetzt, es kommt zum eunuchoiden Hochwuchs. Charakteristisch hierfür sind in Relation zum Rumpf lange Extremitäten, da vor allem die Epiphysenfugen der langen Röhrenknochen betroffen sind. Umgekehrt haben Mädchen mit prämaturer Pubertät (Pubertätszeichen vor dem achten Geburtstag) zunächst ein beschleunigtes Wachstum, aufgrund der zu früh zu hohen Östrogenspiegel und des zu frühen Menarcheneintritts jedoch langfristig ein nicht voll ausgenutztes Wachstumspotenzial.

In der Wachstumsphase wird die Reife des Knochens mittels Röntgenaufnahme der linken Hand beurteilt.
Während des Wachstums nimmt nicht nur die Länge der Knochen zu, er wird auch zunehmend mineralisiert.

Die Peak Bone Mass, die maximale Knochenmasse, wird je nach Definition schon vor dem 20. Lebensjahr oder spätestens mit 30 Jahren erreicht. Während im proximalen Femur die maximale Knochendichte bereits vor dem 20. Geburtstag gemessen wird, ist die Gesamtdichte aller Knochen zum Ende der zweiten Lebensdekade am höchsten [4]. Die maximal zu erreichende Knochenmasse und -dichte ist individuell unterschiedlich und zum größten Teil genetisch determiniert. Treten vor Erreichen der Peak Bone Mass Mangelerscheinungen oder Erkrankungen auf, die zu einer verminderten Knochenmasse führen, sind die Betroffenen besonders gefährdet, später an Osteoporose zu erkranken.

Nach Erreichen der Peak Bone Mass bleibt die Knochendichte über das Erwachsenenalter konstant, es herrscht ein Gleichgewicht zwischen Knochenabund -aufbau, vorausgesetzt die Nährstoffzufuhr und das Körpergewicht bleiben weitestgehend stabil. Ein häufiger Befund ist die verminderte Knochendichte bei jungen Frauen mit Unterernährung und sekundärer Amenorrhö. Bei Frauen unterliegt der Knochenumbau zusätzlich zyklusabhängigen Hormonschwankungen und Veränderungen in Schwangerschaft und Stillzeit. Dass die Knochendichte und Knochenmasse über das Erwachsenenleben stabil bleibt, bedeutet jedoch keineswegs einen $\mathrm{Ru}$ hezustand. Vielmehr handelt es sich um ein ausgewogenes Zusammenspiel der Osteoblasten und Osteoklasten. Diese regulieren sich gegenseitig durch parakrine und autokrine Mechanismen und 
werden durch Hormone und Nährstoffangebot beeinflusst [5].

Östrogen verbessert die intestinale Sensitivität auf aktiviertes Vitamin D und fördert dadurch indirekt die Kalziumresorption. In einer Östrogenmangelsituation wie der Postmenopause wird somit weniger Kalzium aus der Nahrung resorbiert.

Mit zunehmendem Alter überwiegt der Knochenabbau [5]. Dieser ist vor allem bei Frauen in der Menopause zu beobachten, aber auch Männer büßen in etwa ab dem 55. Lebensjahr Knochenmasse ein, was einerseits den sinkenden Sexualsteroidspiegeln und andererseits altersbedingten Stoffwechselveränderungen und geringerer körperlicher Aktivität geschuldet ist [6].

\section{Parathormon (PTH)}

Der Kalzium- und Phosphatstoffwechsel unterliegt der strengen Kontrolle des Parathormons. Es wird in der Nebenschilddrüse gebildet und zählt zu den Peptidhormonen. Es reguliert den Kalzium- und Phosphatspiegel im Blut über Mobilisation aus Knochen sowie Steuerung der Phosphatausscheidung und Kalziumrückresorption in der Niere. Außerdem steigert es die Vitamin-D-Produktion und Aktivierung und somit auch die intestinale Kalziumaufnahme. Rückkoppelnd hemmt das Vitamin D wiederum die PTH-Freisetzung. Hohe Spiegel des PTH führen also zum Anstieg des Kalziumspiegels. Die Ausschüttung des PTH wird durch Kalziumrezeptoren in den Nebenschilddrüsenzellen gesteuert. Bei primärem oder sekundärem Hypoparathyreoidismus muss es substituiert werden, um den Kalziumspiegel aufrechtzuerhalten. Somit ist PTH unabdingbar für den Knochenauf- und -abbau über die Regulation der Kalziumverfügbarkeit. Auch bei der Knochenformierung und -zusammensetzung scheint es beteiligt zu sein. Als Medikament kommt es daher auch bei Osteoporose zum Einsatz [7].

\section{Primärer Hyperpara- thyreoidismus}

Der primäre Hyperparathyreoidismus ist nach Diabetes mellitus und den Schilddrüsenerkrankungen die dritthäufigste endokrinologische Erkrankung mit einer Prävalenz von $0,2-0,4 \%$. Frauen sind zwei- bis dreimal häufiger betroffen, die Diagnose wird meist nach dem 40. Lebensjahr gestellt.

Es handelt sich hierbei um eine sporadisch oder familiär auftretende chronische Übersekretion von PTH. Diese ist durch eine Autonomie der Nebenschilddrüse bedingt. Durch die hohen Spiegel an PTH wird vermehrt Knochen resorbiert. Zusammen mit erhöhter renaler Kalziumresorption entsteht eine Hyperkalzämie. Klassische Symptome sind neben gastrointestinalen Beschwerden Muskelschwäche, Blutdruckerhöhung, Polydipsie, Polyurie sowie Nephrolithiasis und erhöhtes Frakturrisiko/Osteoporose. Es kann aber auch eine asymptomatische Hyperkalzämie vorliegen.

Zur Diagnosestellung führt ein erhöhtes PTH bei erhöhten Kalziumwerten. Eine Bildgebung (Sonografie, Szintigrafie, Cholin-PET, eventuell CT oder MRT) wird zur Lokalisation des Adenoms durchgeführt.

Die Therapie des primären Hyperparathyreoidismus besteht in erster Linie aus der chirurgischen Entfernung des Nebenschilddrüsenadenoms, eine $\mathrm{Ob}$ servanz oder allenfalls Kalzimimetika müssen auf Einzelfallbasis entschieden werden [8].

\section{Sekundärer Hyperpara- thyreoidismus}

Bei Nierenerkrankungen mit erhöhtem Verlust an Kalzium und verminderter Ausscheidung von Phosphat sowie unzureichender Produktion von Calcitriol wird PTH reflektorisch gesteigert, es kommt zum renalen sekundären Hyperparathyreoidismus. Durch vermehrte Freisetzung von Kalzium und Phosphat aus dem Knochen führt dies unbehandelt zur renalen Osteopathie, die durch einen pathologischen Knochenumbau definiert ist und je nach
Dauer der Erkrankung und PTH-Spiegel $\mathrm{zu}$ abnormaler Mineralisation, erhöhtem Knochenvolumen und zur typischen Osteoitis fibrosa cystica führt.

$\mathrm{Ab}$ einer Nierenfunktionseinschränkung von $50 \%$ (glomeruläre Filtrationsrate $<60 \mathrm{ml} / \mathrm{min} / 1,75 \mathrm{~m}^{2}$ ) sind erhöhte PTH-Werte zu beobachten, und es muss mit einer Auswirkung auf die Knochensubstanz gerechnet werden. Die Diagnose der renalen Osteopathie wird mittels Serummarker (Kalzium, PTH, Phosphat und alkalische Phosphatase) oder allenfalls mittels Biopsie gestellt.

Die Therapie zielt darauf ab, die Knochenqualität wiederherzustellen und durch erhöhtes Kalzium entstandene extraskelettale Verkalkungen einzudämmen. Zum Einsatz kommen Phosphatbinder, Kalzimimetika, Vitamin D, Dialyse und auch die Parathyreoidektomie [9].

\section{Calcitonin}

Das Peptidhormon der Schilddrüse fungiert als Gegenspieler des Parathormons. Es entfaltet seine Wirkung durch Bindung an seine Rezeptoren der Osteoklasten und des distalen Nephrons. Es senkt den Kalziumspiegel durch Verminderung der Resorption aus Knochen und Rückresorption in der Niere. Eine ähnliche Substanz, Calcitonin Gene-Related Peptide (CGRP), ein Neuropeptid, ist ebenso an der Aufrechterhaltung des Knochenstoffwechsels durch Regulation der Osteoblastenfunktion beteiligt [10, 11].

Calcitonin hemmt den Knochenabbau und wirkt sich positiv bei Knochenschmerzen bei akuten vertebralen Frakturen aus [11].

\section{Leptin}

Leptin ist ein Peptidhormon, das von Adipozyten produziert wird, deshalb auch Adipokin genannt. Seine Serumkonzentration korreliert mit der absoluten Fettmasse. Es fungiert als Appetitregulator und neuroendokriner Botenstoff. Auf hypothalamischer Ebene beeinflusst es die Freisetzung von GonadotropinReleasing-Hormon (GNRH), Thyreotropin-Releasing-Hormon (TRH), So- 
matotropin (GH) und CorticotropinReleasing-Hormon (CTRH). Das Körpergewicht korreliert positiv mit der Knochendichte. Unabhängig von der Art der „weichen Körpermasse“ (Muskel, Fett, andere Weichteile) ist dieser positive Effekt nachzuweisen.

Der Effekt des Leptins auf die Knochendichte erfolgt einerseits indirekt durch Aufrechterhaltung des Körpergewichts, indem es den Appetit reguliert. Andererseits wirkt es direkt am Knochen durch Bindung an Leptinrezeptoren von Osteoblasten und Chondrozyten. Leptin wird auch von Fettzellen des Knochenmarks produziert, wo es vermutlich direkt Einfluss auf den Knochen nimmt $[12,13]$.

\section{Kortison}

Der Einfluss des Kortisons und anderer adrenaler Steroidhormone auf die Knochendichte wird vor allem in Situationen sichtbar, in denen ein Überangebot herrscht. Beim Morbus Cushing nimmt die Knochendichte deutlich ab. Auch iatrogen erhöhte Kortisonspiegel durch exogene Zufuhr führen zur Abnahme der Knochendichte und einem deutlich erhöhten Frakturrisiko bzw. bei längerer Dauer zur glukokortikoidinduzierten Osteoporose (GIO). Diese Wirkung wird vor allem durch direkte Effekte auf Osteoblasten vermittelt, die bei hohen Kortisonspiegeln ihre Aktivität vermindern und apoptotisch werden. Die Aktivität von Osteoklasten hingegen wird durch Kortison gesteigert [14].

\section{Schilddrüse}

Schilddrüsenhormone entfalten ihre Wirkung auch an Knochen und Knorpel. Veränderungen der Schilddrüsenfunktion beeinflussen den Knochenstoffwechsel. Zum Ausdruck kommt dies besonders in der Wachstumsphase. Mausstudien weisen darauf hin, dass das thyreoideastimulierende Hormon (TSH) den Knochenumsatz hemmt. Kongenitale oder früh auftretende Hypothyreose führt $\mathrm{zu}$ verzögertem Knochenwachstum und Kleinwuchs. Hyperthyreose im Kindesalter führt zu beschleunigtem Knochenwachstum, Kraniosynostose,

J. Klin. Endokrinol. Stoffw. 2021 · 14:48-54 https://doi.org/10.1007/s41969-021-00134-z (c) Der/die Autor(en) 2021

\section{S. Feigl \\ Hormone und Knochenstoffwechsel}

\section{Zusammenfassung}

Der Knochenstoffwechsel ist ein komplexer Vorgang, der von zahlreichen Hormonen abhängt und durch ihre Wirkungen zeitlebens dynamisch beeinflusst wird. Nicht nur in der Zeit des Wachstums und in Hormonmangelphasen, sondern auch in den vielen Jahren des „stabilen Gleichgewichts" im Erwachsenenalter sollte bei verminderter Knochendichte an hormonelle Veränderungen gedacht und vor allem auch eine bestehende Medikation des/der Patienten/in erfragt werden. Aufklärungsarbeit ist besonders wichtig in den kritischen Phasen der Pubertät und Menopause, wo die Verunsicherung der Patientinnen groß und der Einfluss der verschriebenen Hormonpräparate nicht zu unterschätzen ist.

Der Knochenstoffwechsel wird von vielen Hormonen beeinflusst. In der Wachstumsphase ist Somatotropin und Östrogen bestimmend. Parathormon, Calcitonin und Leptin nehmen ebenso eine wichtige Rolle ein. Sie entfalten ihre Wirkung direkt durch Beeinflussung der Osteoblasten und Osteoklasten, aber auch indirekt durch den Vitamin-D-Stoffwechsel und Kontrolle des Körpergewichts. Ist ein Regelkreis gestört, büßen die Betroffenen Knochendichte ein, in jungen Jahren kann es zu vermindertem Körperwachstum kommen Vor allem in der kritischen Phase vor Erreichen der Peak Bone Mass und in der Menopause ist die Funktion der Sexualhormone nicht zu unterschätzen. Besonderes Augenmerk liegt auf dem Einfluss der Kontrazeptiva und der Hormonersatztherapie, welche die Knochengesundheit maßgeblich beeinflussen können.

\section{Schlüsselwörter}

Hormone - Knochenstoffwechsel · Hormonelle Kontrazeption - Menopause Hormonersatztherapie · Hormonwirkung · Schwangerschaft · Stillzeit

\section{Hormones and bone metabolism}

\section{Abstract}

Bone metabolism is a complex process that depends on numerous hormones and is dynamically influenced by their effects throughout life. Not only during growth and hormone deficiency phases, but also during the many years of "stable equilibrium" in adulthood, hormonal changes should be considered in case of reduced bone density and, above all, the patient's existing medication should be requested. Educational work is particularly important in the critical phases of puberty and menopause, when there is great uncertainty among patients and the influence of the prescribed hormone preparations should not be underestimated. Bone metabolism depends on many different hormones. During adolescence growth hormone and estrogen are the key players. Parathyroid hormone, calcitonin, and leptin also play an important role. Hormones directly influence osteoclast and osteoblast function, but also maintain bone density by regulation of vitamin $\mathrm{D}$ metabolism and body weight. In the case of hormonal imbalance, patients suffer from bone density loss and, during adolescence, impaired growth. In particular, prior to achieving the peak bone mass and during the menopausal transition, sex hormones are pivotal. Hormonal contraception and menopausal hormone therapy influence bone density and should be prescribed following meticulous evaluation of the hormonal situation.

\section{Keywords}

Hormones · Bone metabolism · Hormonal contraception · Menopause - Menopausal hormone therapy - Hormonal effects . Pregnancy $\cdot$ Breastfeeding vorzeitigem Epiphysenschluss und letztendlich Kleinwuchs. Funktionsstörungen im Erwachsenenalter führen ebenso zu einem gestörten Knochenstoffwechsel. Eine Hyperthyreose, auch subklinisch (und insbesondere auch häufig iatrogen durch zu hohe Dosierung), erhöht das Risiko für Frakturen deutlich $[15,16]$. 


\section{Der Knochenstoffwechsel der Frau}

Frauen durchleben viele hormonelle Veränderungen: Pubertät und Menarche, Menstruationszyklen, Schwangerschaften, Stillzeiten und Menopause. Da Östrogen, wie bereits beschrieben, großen Einfluss auf den Knochen ausübt, bleibt dieser von den Veränderungen nicht unberührt (• Abb. 1).

Ob der Zeitpunkt der Menarche Einfluss auf die Knochendichte hat, wird diskutiert. Endgültig ist diese Frage jedoch noch nicht $\mathrm{zu}$ beantworten. Einerseits wurden Zusammenhänge der späteren Menarche mit verminderter Knochendichte von bis $\mathrm{zu} 2,8 \%$ und erhöhtem Osteoporoserisiko berichtet, andererseits konnte dieser Effekt in anderen Studien nicht nachgewiesen werden $[4,17]$.

Der Knochen wird unter anderem durch die zyklischen Hormonschwankungen beeinflusst. Zu Beginn des Zyklus sind höhere Knochenumbaumarker nachzuweisen, auch der Parathormonspiegel ist in der ersten Zyklushälfte höher. Um den Zeitpunkt der Ovulation werden die höchsten Vitamin-D-Spiegel gemessen. Durch den hemmenden Einfluss des Östrogens auf den Knochenumbau sinkt der Kalziumspiegel im Laufe des Zyklus ab, Parathormon steigt durch die negative Rückkoppelung an.

Zyklusstörungen (Anovulation, Amenorrhö) können, vor allem wenn sie län- ger vorliegen und mit einem Östrogenmangel einhergehen, die Knochendichte negativ beeinflussen $[17,18]$.

\section{Schwangerschaft und Stillzeit}

Während Schwangerschaft und Stillzeit ist der Kalziumbedarf gesteigert. Dieser liegt während der Schwangerschaft bei ca. $2 \mathrm{~g}$ pro Tag, die Ausscheidung und Aufnahme sind erhöht. Während der Schwangerschaft ist die Bilanz meist ausgeglichen oder leicht positiv. In der Stillzeit werden 200-400 mg Kalzium durch die Muttermilch täglich an den Säugling abgegeben. Um dem hohen Bedarf gerecht zu werden, wird Knochensubstanz resorbiert, während sich die Aufnahme und Ausscheidung wieder auf die Ausgangssituation vor der Schwangerschaft einpendeln [5].

Es resultiert eine Knochendichteabnahme während der Stillzeit, vor allem in den ersten 3-6 Monaten. Hier kommt es zu Verminderung des Mineralgehalts um bis zu $5 \%$, abhängig von der Stilldauer.

Schwangerschaft und Stillzeit haben Studien zufolge jedoch keinen nachhaltigen Einfluss auf die Knochendichte, unabhängig von der Anzahl der Schwangerschaften und der gesamten Stilldauer [17, 19, 20].

\section{Menopause}

Wenn in der Phase der menopausalen Transition der Östrogenspiegel sinkt, nehmen die genannten positiven Effekte des Östrogens ab und das Gleichgewicht des Knochenstoffwechsels verschiebt sich in Richtung Osteoklastenaktivität, die Substanz und Knochendichte nehmen ab. Ein weiterer Verdächtiger neben Östrogen in der Causa Knochenverlust in der Menopause ist das follikelstimulierende Hormon (FSH). Es erhöht die Resorptionstätigkeit der Osteoklasten. Diesem Verlust an Knochendichte und -substanz gehen der Östrogenmangel, FSH-Antwort und der Anstieg an Knochenumbaumarkern voraus. Der größte Verlust an Knochenmasse ist bereits ein Jahr vor und in den zwei Jahren nach der Menopause zu beobachten (Phase der „menopausalen Transition“). Durchschnittlich nimmt die Knochendichte der Lendenwirbel um 2,5\%, die des Femurhalses um $1,8 \%$ in dieser Zeit ab. Die hormonellen Veränderungen sind in dieser Phase auch am stärksten. Der Einfluss auf die Knochendichte korreliert mit dem Ausmaß der Hormonschwankung. Eine Verdoppelung des FSH-Werts resultiert in einer um $0,3 \%$ geringeren Knochendichte pro Jahr [21, 22].

Das durchschnittliche Menopausenalter (definiert als der Zeitpunkt ein Jahr nach der letzten Regelblutung) ist 52 Jahre. Bei einer Lebenserwartung von über

Hier steht eine Anzeige. 


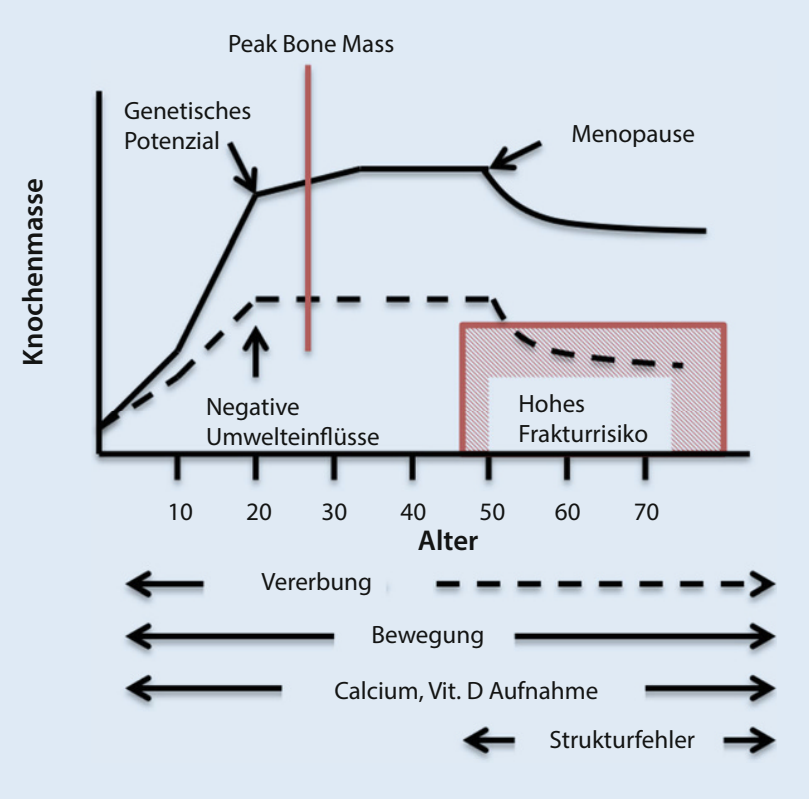

Abb. $1 \varangle$ Vergleich der Zu- und Abnahme der weiblichen Knochendichte und Erreichen der Peak Bone Mass je nach Ausschöpfung des genetischen Potenzials. (Modifiziert nach Heaney et al. [4])

80 Jahren sind Frauen mehr als 30 Jahre lang mit der Abnahme der Knochendichte konfrontiert, insgesamt erkranken bis zu $40 \%$ aller postmenopausalen Frauen an Osteoporose [5].

\section{Therapie in der Menopause}

Inwieweit das Osteoporoserisiko durch Zufuhr exogener Hormone in Form einer Hormonersatztherapie (HRT) vermindert werden kann, ist Gegenstand zahlreicher Untersuchungen.

Eine Hormonersatztherapie gleicht den Östrogenmangel aus, senkt den FSH-Spiegel und führt zu einer signifikanten Erniedrigung des Risikos für Osteoporose-assoziierte Frakturen [23]. Durch zugeführtes Östrogen bleibt das Gleichgewicht zwischen Osteoblasten und Osteoklasten erhalten. Laut der AWMF-Leitlinie „Peri- und Postmenopause - Diagnostik und Interventionen“ ist eine Hormonersatztherapie nicht als alleinige Prävention einer Osteoporose $\mathrm{zu}$ verordnen. Laut Osteoporose-Leitlinie des Dachverbands für Osteologie (DVO) sind Östrogene zur Prävention einer Osteoporose bei postmenopausalen Frauen mit hohem Frakturrisiko zugelassen, wenn eine Unverträglichkeit gegenüber anderer zugelassener Arzneimittel vorliegt.

Eine Indikation für eine HRT ist gegeben, wenn klimakterische Beschwer- den vorliegen, oder als Alternative für eine Osteoporosetherapie, wenn andere Therapien schlecht vertragen werden oder kontraindiziert sind. Die Kombination einer niedrig dosierten Hormontherapie mit einem spezifischen Osteoporosepräparat kann bei klimakterischen Beschwerden und Vorliegen einer Osteoporose erfolgen [16].

Die bei postmenopausalen Frauen mit Osteoporose eingesetzten selektiven Östrogenrezeptormodulatoren (SERM) ahmen die Östrogenwirkung am Knochen nach. Durch ihre agonistische Wirkung in anderen Geweben können klimakterische Beschwerden dadurch jedoch verstärkt werden.

\section{Hormonelle Verhütung}

Hormonelle Kontrazeptiva sind wirksam und beliebt, die Gesamtanwendungsdauer steigt. Es ist unabdinglich, die Langzeitwirkungen zu beleuchten und die Frauen entsprechend aufzuklären.

\section{Gestagenpräparate}

Gestagene sind bewährte und beliebte Verhütungsmittel. Sie sind als Pille, Implantat, Spirale und Injektion verfügbar. Bei Depot-Injektionspräparaten ist Vorsicht geboten. Sie führen zur Erniedrigung des Östrogenspiegels und dadurch zur Verminderung der Knochendichte.
Dieser Effekt ist umso stärker, je länger das Präparat angewendet wird. Nach Absetzen nimmt die Knochendichte wieder zu. Ob nun Frauen, die in jungen Jahren bereits Depot-Gestagene („Drei-Monats-Spritze“) erhalten, auch lange Zeit nach Absetzen nur eine niedrigere Peak Bone Mass erreichen, ist nicht eindeutig geklärt. Auch das Osteoporoserisiko kann hier nicht abgeschätzt werden. In einer 2015 publizierten Cochrane-Metaanalyse wird betont, dass die Knochendichte unter Injektionsgestagenen zwar abnimmt, dies jedoch für Frauen zwischen 18 und 45 keine Einschränkung der Anwendung ergeben sollte [24]. Die AWMF-Leitlinie zu hormoneller Kontrazeption fasst zusammen, dass der Verlust an Knochendichte und -substanz, der unter Injektionsgestagenen besteht, in den meisten Fällen reversibel ist. Eine Anwendung bei Minderjährigen und Frauen mit erhöhtem Osteoporoserisiko sollte sehr zurückhaltend erfolgen und die Anwendung nicht länger als zwei Jahre dauern. Unter Langzeitanwendung sind bei allen Anwenderinnen regelmäßige Knochendichtemessungen angezeigt [25]. Wird das unter Gestagenbehandlung verminderte Östrogen substituiert, kann dem Knochendichteverlust entgegengewirkt werden, wie unter anderem in der Studie von Cromer et al. durch den Vergleich von Östrogen- und Placeboinjektionen gezeigt werden konnte [26]. Zu Gestagenimplantaten fehlen Langzeitdaten, bisher gibt es keine Hinweise auf signifikante Abnahme der Knochendichte unter ihrer Anwendung. Für reine Gestagentabletten wurde kein nachteiliger Effekt auf den Knochen nachgewiesen.

Ein Vergleich zwischen unterschiedlichen Gestagenbestandteilen in oralen Kontrazeptiva zeigte bei Gai et al. keine signifikanten Unterschiede in der Knochendichte nach der Anwendung für 12 und 24 Monate [27].

\section{Kombinierte orale Kontra- zeptiva}

Der Effekt kombinierter oraler Kontrazeptiva auf den Knochen wird in der Cochrane-Analyse als gering eingestuft. Dieser äußert sich vor allem in der Abnahme der Knochendichte bei jungen 
Frauen, die eine Ethinylestradiol-Dosis von 20 Mikrogramm erhielten. Insbesondere unter 30 Jahren scheint die Östrogendosis einen Effekt auf die Knochendichte zu haben, wobei ab einer Dosis von 30 Mikrogramm die Veränderungen klein sind. Über 30 Jahren und in der Prämenopause nimmt dieser Effekt laut einigen Studien ab $[28,29]$. Bei der Verschreibung der Pille für Jugendliche ist es sinnvoll, 30 Mikrogramm Östrogen nicht zu unterschreiten. Für Verhütungspflaster und Vaginalring zeigten sich keine wesentlichen Effekte auf die Knochendichte [30]. Zusammenfassend sind die Aussagen der Studien mäßig stark aufgrund der fehlenden Möglichkeit der Placebokontrolle bei Kontrazeptionsmethoden. Eine klare Aussage über das Frakturrisiko kann außerdem nicht getroffen werden, da randomisiert-kontrollierte Studien fehlen.

\section{Natürliches Östrogen versus Ethinylestradiol}

Für junge Frauen mit prämaturer Ovarialinsuffizienz (Versagen der Ovarialfunktion vor dem 40. Lebensjahr) ist die Substitution mit natürlichem Östrogen zur Prävention der Osteoporose gegenüber dem künstlichen Ethinylestradiol zu bevorzugen. Es scheint eine bessere protektive Wirkung auf den Knochen zu haben [31].

\section{Fazit für die Praxis}

Störungen des Hormonhaushaltes
im Kindes- und Jugendalter beein-
flussen die Knochendichte und das
Körperwachstum nachhaltig. Bei
vermindertem oder beschleunigtem
Körperwachstum und zu frühen oder
späten Pubertätszeichen müssen
Hormonstörungen abgeklärt wer-
den. Die Peak Bone Mass wird mit
20-30 Jahren erreicht. Zu niedrig
dosierte kombinierte Pillen und
Injektionsgestagene können die
Knochendichte negativ beeinflussen
und sollten bei jungen Mädchen
zurückhaltend angewendet werden.
Parathormon, Leptin, Schilddrüsen-
hormone und Kortikosteroide beein-
flussen die Knochendichte und das

Frakturrisiko stark. Um die Menopause nimmt die Knochendichte signifikant ab, eine Hormonersatztherapie wirkt diesem Effekt entgegen. Sie sollte jedoch nicht zur alleinigen Prävention der Osteoporose verordnet werden, sondern nur, wenn klimakterische Beschwerden vorliegen, oder als Alternative, wenn keine anderen Osteoporosetherapeutika vertragen werden.

\section{Korrespondenzadresse}

\section{Dr. med. univ. Sarah Feig}

Universitätsklinik für Frauenheilkunde und Geburtshilfe, Medizinische Universität Graz Auenbruggerplatz 14, $8036 \mathrm{Graz}$, Österreich s.feigl@medunigraz.at

Funding. Open access funding provided by Medical University of Graz.

\section{Einhaltung ethischer Richtlinien}

Interessenkonflikt. S. Feigl gibt an, dass kein Interessenkonflikt besteht.

Für diesen Beitrag wurden von der Autorin keine Studien an Menschen oder Tieren durchgeführt. Für die aufgeführten Studien gelten die jeweils dort angegebenen ethischen Richtlinien.

Open Access. Dieser Artikel wird unter der Creative Commons Namensnennung 4.0 International Lizenz veröffentlicht, welche die Nutzung, Vervielfältigung, Bearbeitung, Verbreitung und Wiedergabe in jeglichem Medium und Format erlaubt, sofern Sie den/die ursprünglichen Autor(en) und die Quelle ordnungsgemäß nennen, einen Link zur Creative Commons Lizenz beifügen und angeben, ob Änderungen vorgenommen wurden.

Die in diesem Artikel enthaltenen Bilder und sonstiges Drittmaterial unterliegen ebenfalls der genannten Creative Commons Lizenz, sofern sich aus der Abbildungslegende nichts anderes ergibt. Sofern das betreffende Material nicht unter der genannten Creative Commons Lizenz steht und die betreffende Handlung nicht nach gesetzlichen Vorschriften erlaubt ist, ist für die oben aufgeführten Weiterverwendungen des Materials die Einwilligung des jeweiligen Rechteinhabers einzuholen.

Weitere Details zur Lizenz entnehmen Sie bitte der Lizenzinformation auf http://creativecommons.org/ licenses/by/4.0/deed.de.

\section{Literatur}

1. Blumsohn A, Hannon RA, Wrate R et al (1994) Biochemical markers of bone turnover in girls during puberty. Clin Endocrinol 40(5):663-670
2. van Coeverden SC, Netelenbos JC, de Ridder CM et al (2002) Bone metabolism markers and bone mass in healthy pubertal boys and girls. Clin Endocrinol 57(1):107-116

3. Tanner JM, Whitehouse RH, Marshall WA et al (1975) Prediction of adultheight from height, bone age, and occurrence of menarche, at ages 4 to 16 with allowance for midparent height. Arch Dis Child 50(1):14-26

4. HeaneyRP,AbramsS, Dawson-HughesBetal(2000) Peak bone mass. Osteoporos Int 11(12):985-1009 5. Leidenberger S (2014) Ortmann. Klinische Endokrinologie für Frauenärzte. Springer, Berlin Heidelberg

6. Manolagas SC (2010) From estrogen-centric to aging and oxidative stress: a revised perspective of the pathogenesis of osteoporosis. Endocr Rev 31(3):266-300

7. Goltzman D (2018) Physiology of parathyroid hormone. Endocrinol Metab Clin North Am 47(4):743-758

8. AWMF. S1-Leitlinie - Primärer Hyperparathyreoidismus. Leitlinie der Deutschen Gesellschaft für Kinderendokrinologie und-diabetologie (DGKED) e.V.2016.

9. Endokrinologie DGf. Rationelle Diagnostik und Therapie in Endokrinologie, Diabetologie und Stoffwechsel Thieme; 2010.

10. de Paula FJ, Rosen CJ (2010) Back to the future: revisiting parathyroid hormone and calcitonin control of bone remodeling. Horm Metab Res 42(5):299-306

11. Lerner UH (2006) Deletions of genes encoding calcitonin/alpha-CGRP, amylin and calcitonin receptor have given new and unexpected insights into the function of calcitonin receptors and calcitonin receptor-like receptors in bone. JMusculoskelet Neuronal Interact 6(1):87-95

12. Reid IR, Baldock PA, Cornish J (2018) Effects of Leptin on the skeleton. Endocr Rev 39(6):938-959

13. Upadhyay J, Farr OM, Mantzoros CS (2015) The role of leptin in regulating bone metabolism. Metabolism 64(1):105-113

14. Hardy R, Cooper MS (2010) Adrenal gland and bone. Arch Biochem Biophys 503(1):137-145

15. Gogakos Al, Bassett DJH, Williams GR (2010) Thyroid and bone. Arch Biochem Biophys 503(1):129-136

16. DVO. Leitlinie: Prophylaxe, Diagnostik und The rapie der Osteoporose bei postmenopausalen Frauen und bei Männern. 2017.

17. Waugh EJ, Lam MA, Hawker GA et al (2009) Risk factors for low bone mass in healthy 40-60 year old women: a systematic review of the literature. Osteoporos Int 20(1):1-21

18. Ouyang F, Wang X, Arguelles L et al (2007) Menstrual cycle lengths and bone mineral density: a cross-sectional, population-based study in rural Chinese women ages 30-49 years. Osteoporos Int 18(2):221-233

19. Cooke-Hubley S, Gao Z, Mugford G et al (2019) Parity and lactation are not associated with incident fragility fractures or radiographic vertebral fractures over 16 years of follow-up: Canadian Multicentre Osteoporosis Study (CaMos). Arch Osteoporos 14(1):49

20. Kovacs CS (2016) Maternal mineral and bone metabolism during pregnancy, lactation, and post-weaning recovery. Physiol Rev 96(2):449-547

21. Karlamangla AS, Burnett-Bowie SM, Crandall CJ (2018) Bone health during the menopause transition and beyond. Obstet Gynecol Clin North Am 45(4):695-708 


\section{Originalien}

22. Crandall CJ, Tseng CH, Karlamangla AS et al (2013) Serum sex steroid levels and longitudinal changes in bone density in relation to the final menstrual period. JClin Endocrinol Metab 98(4):E654-E663

23. AWMF. S3 Leitlinie: Peri- und Postmenopause Diagnostik und Interventionen. 2020.

24. Lopez LM, Chen M, Mullins LS et al (2015) Steroidal contraceptives and bone fractures in women: evidence from observational studies. Cochrane Database Syst Rev. https://doi.org/10. 1002/14651858.CD009849.pub3

25. AWMF. S3 Leitlinie Hormonelle Empfängnisverhütung. 2020.

26. Cromer BA, Lazebnik R, Rome E et al (2005) Double-blinded randomized controlled trial of estrogen supplementation in adolescent girls who receive depot medroxyprogesterone acetate for contraception. Am J Obstet Gynecol 192(1):42-47

27. Gai L, Jia Y, Zhang M et al (2012) Effect of two kinds of different combined oral contraceptives use on bone mineral density in adolescent women Contraception 86(4):332-336

28. Herrmann M, Seibel MJ (2010) The effects of hormonal contraceptives on bone turnover markers and bone health. Clin Endocrinol 72(5):571-583

29. Warholm L, Petersen KR, Ravn P (2012) Combined oral contraceptives' influence on weight, body composition, height, and bone mineral density in girlsyoungerthan 18years:a systematic review. Eur J Contracept Reprod Health Care 17(4):245-253

30. Massaro M, Di Carlo C, Gargano V et al (2010) Effects of the contraceptive patch and the vaginal ring on bone metabolism and bone mineral density: a prospective, controlled, randomized study. Contraception 81(3):209-214

31. Webber L, Davies M, Anderson R et al (2016) ESHRE Guideline: management of women with premature ovarian insufficiency. Hum Reprod 31(5):926-937

Hinweis des Verlags. Der Verlag bleibt in Hinblick auf geografische Zuordnungen und Gebietsbezeichnungen in veröffentlichten Karten und Institutsadressen neutral.

Hier steht eine Anzeige.

\section{Springer}

